\title{
Implications of document-level literacy skills for Web site design
}

\author{
MARY ANNE BRITT \\ Northern Illinois University, DeKalb, Illinois \\ and \\ GARETH GABRYS \\ Apropos Technology, Oakbrook Terrace, Illinois
}

\begin{abstract}
The proliferation of World Wide Web (Web) sites and the low cost of publishing information on the Web have placed a tremendous amount of information at the fingertips of millions of people. Although most of this information is at least intended to be accurate, there is much that is rumor, innuendo, urban legend, and outright falsehood. This raises problems especially for students (of all ages) trying to do research or learn about some topic. Finding accurate, credible information requires documentlevel literacy skills, such as integration, sourcing, corroboration, and search. This paper discusses these skills and offers a list of simple ways that designers of educational Web sites can help their visitors utilize these skills.
\end{abstract}

In many ways, the World Wide Web (Web) is an ideal educational tool. By typing a few keywords and clicking, one can instantly find information on virtually any topic. Unfortunately, this ease of access comes with a price. Although much of the information on the Web is at least intended to be accurate and helpful, there is also much that is speculation, anecdote, urban legend, or outright deception. Students are thus faced with the task of discriminating between those sources of information that are credible and those that are not. Although one would hope that students will acquire the skills they need to reason about the documents they encounter, our review of document-level literacy skills in the next section shows that this is not always the case. Our focus elsewhere has been on how to instruct students in the use of these skills (Britt \& Gabrys, 2001; Britt, Perfetti, Van Dyke, \& Gabrys, 2000); here, we turn our attention to how Web site design can also play a role. Although it is clearly not the responsibility of content providers to teach document-level literacy skills, there are nevertheless some fairly simple things that they can do in the design of their sites to assist students in the use of these skills.

\section{DOCUMENT-LEVEL LITERACY SKILLS}

Document-level literacy skills are ways of thinking and reasoning about documents. To a certain extent, people use these skills automatically all the time. Unfortunately, their use is often inconsistent and inadequate. The

Correspondence concerning this article should be addressed to M. A. Britt, Psychology Department, Northern Illinois University, DeKalb, IL 60115 (e-mail: britt@niu.edu). first author's research on learning from multiple documents (Britt, Perfetti, Sandak, \& Rouet, 1999; Perfetti, Rouet, \& Britt, 1999) has focused on four skills: integration, sourcing, collaboration, and to a lesser extent, search. In this section, we will describe each of these skills, briefly relate studies of students' competence in each skill, and finally discuss why each skill is important to Web users.

\section{Integration}

Integration is the construction of a mental representation of a situation that consolidates new information with information that one already knows. Although integration goes on all the time, it is especially important in cases in which people learn by reading multiple documents on a single topic. With printed linear texts (e.g., books), the author of the text assists the reader by giving a single, consistent, and coherent presentation of the material. Authors facilitate understanding by presenting the concepts or events in a meaningful order and reminding the reader of background knowledge that is necessary for comprehension. When learning from multiple texts, especially nonlinear ones (e.g., Web pages), the reader assumes a greater burden-activating relevant prior knowledge, filling in gaps, and reconciling discrepancies. The situation is further complicated when information is acquired at different times and from different authors. Although some prior knowledge is no doubt automatically activated (Myers \& O'Brien, 1998; Myers, O’Brien, Albrecht, \& Mason, 1994), deliberate planning and inference may also be required to seek out necessary information and resolve inconsistencies.

Research suggests that there are limits on people's ability to integrate information from multiple documents. 
College students have difficulty modifying a representation from a previously read text on a topic when given a correction on the same topic (Johnson \& Seifert, 1994, 1999; van Oostendorp \& Bonebakker, 1999). High school students appear to have even more difficulty. Britt, Goldman, and Perfetti (1999) found that high school students who were given two related but opposing texts sequentially, on a single occasion, did not spontaneously integrate information from the texts and generally found it very confusing that there could be texts that presented conflicting perspectives. However, with explicit instructions to integrate and a short explanation of how to do it, these high school students integrated the two texts to a level comparable to that of students reading the same material presented as a single integrated text. This research suggests that although students have difficulty integrating and may not always engage in it spontaneously, they nevertheless can do it when prompted and provided with assistance.

Learning from the Web makes greater demands on a reader's ability to integrate than does normal textbook reading. Web documents are written as stand-alone documents and may be broken up over many short pages. Readers are expected to guide their own learning and can read texts in any order. This puts an added burden on the reader, rather than the author, to establish coherence (Charney, in press; Foltz, 1996). Furthermore, it is not always easy to tell where the work of one author ends and that of another begins. Many perceptual cues, normally available with print documents, are lost on the Web. For example, switching from reading one book to another is a clear perceptual experience involving many features; clicking a link to jump to another Web document is not necessarily such a clear change in context. Unpredictably, the link may be to a different location in the same document, to another page by the same author, or to another page entirely. The sheer quantity of available information exacerbates the problem. The Web has such a low cost of publication that many voices are heard of authors who might not otherwise ever have attempted to be published or who would likely have been denied publication. This tends to increase the amount of inconsistent and conflicting information, placing a greater burden on integration. Now let's turn to the evaluation of the credibility of this information.

\section{Sourcing}

Sourcing is the skill of gathering information about a source and using it to form conclusions about a document, especially conclusions regarding credibility. Wineburg (1991) carried out a detailed analysis of differences between expert and novice readers of multiple documents in history. He found that before experts read a document, they systematically sought out information about the author (e.g., name, credentials, relationship to information they are writing about) and the document (e.g., when and where it was written, document type). He referred to this process of noticing the source prior to learning from it as a sourcing heuristic. We extend the term sourcing to refer to a more general process of identifying, evaluating, and using source information and connecting source information to the document's content. For printed texts, source identification is as simple as reading the book cover, the copyright notice, or the first page of an article to identify the author's name, affiliation, publication date, publisher, other books by the same author, and acknowledgments. Once the reader identifies the source, he or she can use this information, along with the document type, to evaluate the source. In general, this involves a fairly complicated reasoning process, one outcome of which is to assess the credibility of the information presented in the document. With print documents, the reader's task may be aided by the fact that a reputable company published it or that a library chose to acquire it.

As was noted, expert historians routinely engage in sourcing. They spontaneously search for source information even if it is not immediately available, they note a variety of source features, and they weigh features in complex ways during source evaluation (Rouet, Britt, Mason, \& Perfetti, 1996; Rouet, Favart, Britt, \& Perfetti, 1997; Wineburg, 1991). College and advanced placement high school students consider source information under much more limited circumstances and, generally, only when conditions are optimal (Rouet et al., 1996; Rouet et al., 1997; Wineburg, 1991). Although college students do notice several features of a source (Rouet et al., 1996) and do connect that information to at least the central content (Britt et al., 1999), it appears that this occurs only under optimal conditions and that they represent only some of the characteristics of a source, and not always the most important ones. High school students have even more difficulty representing and evaluating source information and usually fail to do so unless given a supportive environment (Britt \& Aglinskas, in press; Britt, Rouet, \& Perfetti, 1996).

The Web presents greater demands on sourcing ability than does printed matter. A student researching a paper in a library will tend to read a relatively small number of articles and books. When the Web is used, however, the ease of searching and retrieving documents may lead a student to search through dozens of sources. Furthermore, the ease of obtaining information about the source is more difficult for Web documents than for printed ones. There are no agreed-upon standards for providing source information on the Web. Upon arriving at a site, the student must locate whatever source information is provided and try to infer other important features of the document and its author. For example, the student must determine the following: Who authored the page? When was it written? When was it last updated? What is the author's affiliation? How does the author know the information he or she writes about? What are the author's motives for writing? The reader may have little to work with when trying to determine whether the information is trustworthy. Sometimes no source infor- 
mation other than the URL is available, and even this might be only an IP address. Even when source information is available, its interpretation is not always straightforward. Normally reputable and mainstream sources of information sometimes have lower criteria for the accuracy of information they make available on the Web, much of the information that is available is really advertising; and information may quickly become out of date. There are also special problems with citing Web documents. In particular, many Web documents get moved, renamed, or deleted, making it difficult to locate them at a later date, and there is, of course, no guarantee that the content of the page itself might not change.

\section{Corroboration}

Corroboration is the skill of seeking out independent confirmation (and possibly disconfirmation) for some information. Wineburg (1991) noted that while reading a document, expert historians check external sources to verify the accuracy of information, an activity he referred to as a corroboration heuristic. This process involves seeking out other relevant information from memory or available texts and determining what information is agreed upon and what is uniquely mentioned only in this document. Printed texts generally provide citations and bibliographic information that can be used to locate the actual documents mentioned by an author to support his or her claims; a reader can use this information to evaluate whether the sources do in fact exist and are represented accurately. Readers can also avail themselves of many other resources, such as published bibliographies and citation indexes, to independently research a topic and search for corroboration.

Evidence suggests that people rarely corroborate the information they read. Wineburg (1991) found that high school students failed even to notice discrepant information, let alone follow up by comparing documents. Presumably, providing students with some support would help them engage in corroboration. For instance, Wiley (2001) found that providing students with two-window environments supports their ability to compare and contrast information across texts and leads to better comprehension and integration of the material in the texts.

Because the Web lowers the bar for publishing and makes it possible to find credible information and apocrypha with equal ease, corroboration must be viewed as an absolutely essential skill for Internet users. The Web abounds with errors, rumors, urban legends, outright falsehoods, and marketing literature. Fortunately, the same search tools that place such questionable material at your fingertips also make it relatively easy to search for corroborating evidence. With several clicks of the mouse, Web users can have immediate access to large bibliographies on the topic area, citation indexes, research databases (such as PsychInfo), archival sites, and search engines. They can also open several windows on the screen simultaneously to facilitate the comparison process. However, simple corroboration is not enough.
Corroboration must be used in conjunction with sourcing. Because erroneous information abounds and circulates, it is frequently possible to find multiple sites that spread the same errors. Thus, one must look not only for independent confirmation, but also for credible independent confirmation. Searching for disconfirming evidence is also sometimes valuable, especially in cases of widely believed fabrications, such as urban legends. Many sites, sometimes even reputable ones, may repeat such stories, often citing legitimate sources for them. Only by exhaustively tracking a citation or by finding an explicit disconfirmation can some legends be properly discredited.

\section{Search}

Search is a skill that is interwoven throughout the other skills and the entire research process. One must use search skills to find relevant documents before integration becomes an issue. One must also use search skills to locate (or fail to locate) cited sources when corroborating. Search requires both awareness of and skill in using specially designed tools. Some of these tools are very general; some are topic specific. Some are easy to use; some require explicit training. Search requires some degree of knowledge about the research topic and some degree of imagination and general problem-solving ability.

A considerable amount of research has been devoted to looking at how individuals construct queries for search engines. In general, these studies have found that people are not particularly skilled at searching (Rouet \& Levonen, 1996). Novices often use very nonspecific queries in search engines that return millions of links to irrelevant Web pages. This results in frustration and failure to locate possible corroborating documents. The selection of appropriate keywords and the construction of Boolean queries are skills that require domain knowledge and a little training. Fang and Salvendy (2000), for instance, showed that providing people with simple information about how frequently their search terms appeared in a search set greatly improved their ability to find relevant documents.

Search is virtually synonymous with Web use. It is hard to imagine doing anything on the Web without using either a directory or a search engine. Other than a library at which keyword search is used, there are not many places where we would learn to perform these kinds of searches. Fortunately, it is not difficult to perform a simple search. In most cases, entering a single keyword is sufficient to locate what we want. It is only when we need to find specific, uncommon information that skill is required to perform the search. Unfortunately, this is often the situation when we search for sources to corroborate some statement.

\section{SUGGESTIONS FOR EDUCATIONAL WEB SITE DESIGNERS}

We will now offer suggestions for Web site designers on how they can make their sites more conducive to the 
use of document-level literacy skills. These suggestions are intended mainly for those who expect to have people, especially students, learn some content while visiting their site. This would include both those with content sites of an educational or archival nature and those offering actual courses. These suggestions, although not intended to be exhaustive, illustrate how design decisions can impact readers. Web site designers may find that they are already following many of these suggestions; indeed, some are even built into software packages that create and manage sites.

\section{Integration}

Our first suggestions are related to helping a reader maintain a consistent context while reading documents that may span several pages. Context is a rather amorphous concept that includes the spatiotemporal characteristics of the reading environment, as well as the reader's goals and the actual content of the documents. Like goals, context has a hierarchical organization. Thus, one must remember that there may be several levels of context to consider. Managing a reader's context involves creating both a sense of continuity related to the reader's overall goal for visiting the site and appropriate shifts of context when certain boundaries are reached.

Suggestion 1: Create a consistent look and feel for the site. One aid to integration is the continuity of the reading experience. Even when content is spread out over numerous pages, one can help the reader to maintain a sense of continuity by creating a consistent look and feel. Visual devices, such as background, color, common headers, footers, and navigation bars can help, along with consistent use of fonts and headings. Most Web site design packages support the use of templates to create a consistent look and feel for a site. Nevertheless, we have seen sites in which overexcited Web site designers have chosen a different background and color scheme for every page on their site. For Web sites that need to present content on the same topic over multiple pages, continuity of look and feel is essential for helping the reader to minimize distraction and maintain context (cf. Suggestion 5).

Suggestion 2: Avoid taking the reader to a new page to display ancillary materials. One of the best ways to preserve context is to not change the page. Researchers have found that there is a cognitive load associated with reorienting oneself after following links (Black, Wright, Black, \& Norman, 1992; Wright, 1991). Thus, when displaying ancillary material, such as definitions, commentary, and examples, use techniques, such as popup windows, frames, or rollover windows, that do not change the main window page. One often sees Help pages for Web applications displayed in popup windows so the user can view the help material while still maintaining the application state. As more users upgrade to browsers that support dynamic HTML, other options will become available, such as dynamically showing and hiding page sections, rollover windows, and so forth.
Suggestion 3: Avoid using too many embedded links in a text. In addition to being visually distracting, embedded links encourage the reader to click on them, thus interrupting the flow of reading. Using embedded links to provide definitions, cross-references, within-page jumps, and additional resources leads to a breakdown in coherence (Wright, 1991) and is frequently distracting. Although some have championed highly interlinked texts as a means for empowering learners to pursue their own nonlinear learning course (Landow, 1989), when the goal is to have someone read documents of more than a few paragraphs in order to learn something, such links are likely to cause a breakdown of coherence. Instead, other devices for breaking up a page, such as a text box or a gloss, may be better suited for providing definitions without requiring the reader to click or (worse) change the page.

That said, it is nevertheless desirable to offer students the opportunity to explore other pages and sites. Referenced materials must be cited within the text, and it is good practice to provide links to these sources when they are available on line. Links to related materials, however, can be taken out of the text and placed together at the end of a document or in a text box. Such links can be annotated to indicate their relationship to the current document. This minimizes the impact on reading, and annotation enables the designer to reinstate the context for the new material. One might argue that situating the link in the text itself gives the reader the opportunity to read the new material while still in the original learning context. This is only partially true, however. First, depending on the placement of the link, the reader may not have finished reading the original text statement and, thus, may lack context. Second, providing an annotated link at the end of the text both creates an opportunity for restating the idea and reinstates the context. A reinstated context is presumably more complete after the reader has finished the text.

Suggestion 4: Minimize distractions. Many Web sites, especially sponsored ones, are intentionally distracting. The content is basically a lure for people to come and view advertising. Many news sites, for example, challenge readers to ignore flashing banner ads, nonscrolling margin ads, popup windows, large pictures inserted into the text that are unrelated to the content, and even graphic sprites that flitter around over the text itself, preventing one from reading. Needless to say, it is difficult for people to read in such an environment and even more difficult to learn something from what they read. Nor is it necessary that the distractions should be unrelated to the site's content. Many textbooks, for instance, provide so many pictures, text boxes with case studies, sidebars, and footnotes that it is a wonder students learn anything from them. In fact, Mayer and his colleagues have found decrements to understanding from texts as a result of such seductive details (Harp \& Mayer, 1998; Mayer, Heiser, \& Lonn, 2001). Although breaking up a long text with illustrations and text boxes can make a text appear less daunting, be careful to avoid 
the tendency of many Web designers to clutter their pages with unnecessary decorations, such as animated GIFs and Wingding fonts.

Suggestion 5: Clearly demarcate document boundaries. The focus of our previous suggestions was on not changing the context while the user is reading. However, there will be times when you will want to make a very clear change in context. One such occasion is when the user switches to a new document. One drawback of creating a consistent look and feel for a site is that it is easy to lose a sense of document boundary. This is not really a problem for sites that present content from a single source (much in the same way as a textbook), but for sites that present content from multiple documents, especially when there are documents from different authors, it is important to clearly mark where one document ends and another begins. Perhaps the strongest way to indicate a change in context is with a page change. Sometimes, however, this is not enough, especially when single documents already span multiple pages. Other indicators, such as headers and changes in font, background, and to a lesser extent, color, may be called upon to create a sense that the context has changed.

It is important to keep in mind that although we may wish to change the context of the document, we do not want to change the context of the reader's goal or topic. Thus, we must balance changes in document context with preserving the overall site and goal contexts. Thus, some general aspects of the site's look and feel should remain the same, whereas others can change to mark a document boundary.

\section{Sourcing and Corroboration}

The key ways to support sourcing and corroboration are to make source information and comparison information easily available.

Suggestion 6: Display source information on all the pages of the site, not just on the home page. One crucial way to support sourcing and corroboration is to clearly identify yourself on all pages of your site. Some sites put the name of their organization only on the home page, so that if a user locates a page with a search engine, the user must either find the home page or infer the source from the URL. Most Web servers now make including a common header file easy through support for server-side includes (SSIs). SSIs are special commands that appear as HTML comments but actually direct the server to include another file when sending a page to a user. By placing such a directive at the beginning of each of your Web pages, you can ensure that standard identifying information is always displayed, without having to copy it to every Web page. To use SSIs, give your Web page a .shtml extension and then use a directive in your page to handle the include. For example, placing the line $<$ !--\#include file="header.htm"--> in your HTML file will cause the contents of the file "header.htm" to be included when someone receives your page. Another way to use standard headers and footers is to use CGI scripts or Microsoft's ASP with VBScript or JScript to construct the HTML page.

Another way in which some sites provide consistent source information and navigation links is through the use of HTML frames. The main advantage of frames is that they enable contextual features, such as banners and navigation links, to remain on the screen while the content page is free to scroll. Unfortunately, frames are plagued with difficulties associated with printing and the handling of which pane has the input focus. Such difficulties have led many designers to avoid their use on sites for which independent scrolling is not essential. One can achieve a similar partitioned layout by using HTML tables and scripting. For example, one could create a "header.html" file that contained a table that organized the page into sections, as follows:

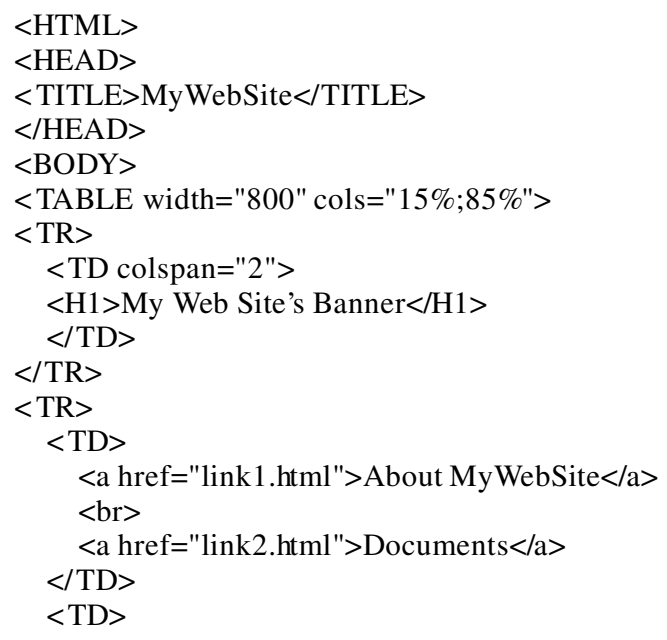

This will display the banner across the top of the page and a series of links in a narrow column along the left side. A "footer.html" file could then simply close the table and the page and optionally display a bottom banner or navigation links, as follows:

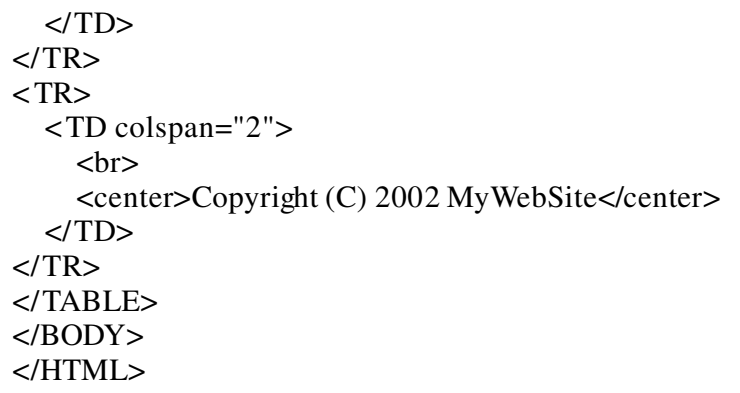

One would then use the "header.html" and "footer.html" files as includes in each content page, as follows:

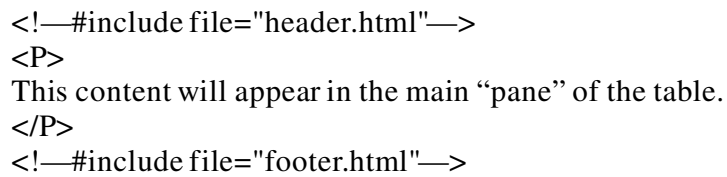


When the content page is loaded, "header.html" sets up the table, displays the banner and left navigation bar, and then the main content of the page is shown; then, "footer.html" closes the table and perhaps displays a bottom banner. This technique enables you to display a consistent look and feel, as well as a common navigation bar, without the drawbacks associated with frames. In general, the use of SSIs enables this consistency to be achieved without needing to duplicate the same settings in multiple files.

Suggestions 7 and 8: Provide extended source information when citing a source; provide links to cited sources when available. When you refer to a source, provide extended source information in your document if it is available. For example, rather than saying "according to CNN," give the reader enough information to find the document (by providing the date it was written or broadcast, the program or correspondent's name, and so forth). If the source is available on line, provide a link to it. Otherwise, provide enough bibliographic information to enable the reader to locate it. Keep in mind that Web documents have a fleeting existence. You may want to quote the critical material you cite, in case it disappears, or keep hard or soft copies of Web documents that you cite, although you should not make such copies available on line without the copyright holder's permission.

Suggestion 9: Look after links you provide. Because Web pages are frequently renamed, moved, or removed, it is important to monitor the links you create on your Web pages. Sending users to invalid Web pages communicates that your information may be old or out of date. Many Web site management tools provide automatic link checking. Use this feature to periodically check your links and repair any broken ones.

Suggestion 10: Provide a list of related and contrasting links at the end of your document. Provide readers with a list of related links at the end of your text. This list can be annotated to explain the relevance of each link, and it can include sites that present both supporting and contrasting views (if applicable). Keep in mind that readers may also infer that the sources you cite are credible because you cited them. You lend your credibility to other sites, so be careful to point out when you use sources that are of questionable credibility.

Suggestion 11: Use consistent and meaningful domain names on your site. Keep in mind that your URL contains source information. Do not reroute visitors on your site to another Web server that does not use the same domain name, especially not to one that has only an IP address. The primary domain name (e.g., yahoo.com) is one of the most reliable pieces of information that is generally available to the user and thus can be used as an authentication check. The contents of a Web site can be easily copied and displayed on another site (witness the numerous pseudo-duplicates of the Yahoo home page), but hijacking a domain name is much more difficult.

\section{Search}

Our final few suggestions relate to search. There are two types of document search: within-text search and between- text search. We are mainly interested in between-text search here. Many studies have been published on both of types of search, and we would refer interested readers to these works. We offer suggestions here that are related to our particular concern with supporting learning from multiple documents. Helping Internet users locate relevant documents includes both helping people to find your site and providing them with links and tools to find other relevant pages on your site and others.

Suggestion 12: Use META elements in your pages with appropriate keywords so search engines can find you. Not every page on your site is likely to contain all of the keywords that are relevant to the general document. One way to overcome this limitation is to use the HTML META element to provide them. The META element is used to provide information about the document that is not displayed on the page. Many search engines look for a special "keywords" attribute and include it in their index keys for the page. For example, inserting the following HTML code will help search engines to associate all of these keywords with your page: <META NAME $=$ "keywords" CONTENT="reading document literacy skills Web-site design">

Suggestion 13: Register the site with general directories (e.g., Yahoo) and with topic-specific directories. One way to help others find your pages is to register with site directories. Some directories are general, such as Yahoo; others are more topic specific. Unless your site is very famous, you will likely need to request to be added.

Suggestion 14: Support others who might create links to your pages. Make sure that your links remain valid. Do not depend on users letting you know if a link does not work. Every time you add, remove, or change a file, assess the impact on those who might be linked to you. If you need to change a page name or directory, add virtual paths on your Web server to ensure that the old reference redirects to the new page. You can also monitor your Web server's log files to check for cases in which legitimate but outdated paths have failed in order to fix them.

Suggestion 15: If appropriate, encourage visitors to add your site to their browser's shortcut list. If you have a site that might be of general use to some people, prompt users to add your page to their browser's list of "favorites." You can even provide a button that will run a script to pop up the browser's dialog for adding favorites.

Suggestion 16: If you have a large site, provide site maps and search capability. For Web sites with many pages, it is helpful to display the structure of the site as a diagram that allows users to jump to specific locations. In addition, it may be helpful to provide keyword search capability.

Suggestion 17: Provide links to topic-specific directories and search engines. Although many sites do so, it is not really necessary to provide links to general search engines. Many users have favorite search engines or use their browser's built in search tools. However, if 
you are aware of topic-specific directories and search engines, it would definitely be helpful to provide links to them.

Suggestion 18: Display keywords that others might use to find related topics. In addition to providing links to related sites, it can sometimes be helpful to provide visitors with keywords they can use to search for related sites. Many users are not very skilled at generating keyword sets that sufficiently narrow their search, so seeing a query might be quite useful to them.

\section{CONCLUSIONS}

We have endeavored to show that the Web has turned everyone into a researcher. Skills that have been the traditional province of high school and college classes on the writing of research papers are now essential for anyone seeking to learn something while Web surfing. Unfortunately, many people never learn or only partially learn these skills in school. The situation is even worse for those whose basic reading skills may be weak. Our hope is that avenues of support will become available for those who need it. Indeed, our own efforts at providing tutoring for document-level literacy skills have been encouraging (Britt \& Gabrys, 2001; Britt et al., 2000). Perhaps, eventually, standards will arise for establishing credibility (or at least authenticity), in the way that they are beginning to arise for protecting privacy. Until that day, however, caveat emptor.

\section{REFERENCES}

Black, A., Wright, P., Black, D., \& Norman, K. (1992). Consulting on-line dictionary information while reading. Hypermedia, 4, 145169.

Britt, M. A., \& AglinsKas, C. (in press). Improving student's ability to identify and use source information. Cognition \& Instruction.

BRITT, M. A., \& GABRYS, G. (2001). Teaching advanced literacy skills for the World Wide Web. In C. R. Wolfe (Ed.), Learning and teaching on the World Wide Web (pp. 73-90). San Diego: Academic Press.

Britt, M. A., Goldman, S. R., \& Perfetti, C. A. (1999, August). Content integration in learning from multiple texts. Paper presented at the Meeting of the Society for Text and Discourse, Vancouver, BC.

Britt, M. A., Perfetti, C. A., Sandak, R, \& Rouet, J.-F. (1999). Content integration and source separation in learning from multiple texts. In S. R. Goldman, A. C. Graesser, \& P. van den Broek (Eds.), Narrative comprehension, causality, and coherence (pp. 209-233). Mahwah, NJ: Erlbaum.

Britt, M. A., Perfetti, C. A., Van Dyke, J., \& Gabrys, G. (2000). The Sourcer's Apprentice: A tool for document-supported history instruction. In P. N. Stearns, P. Seixas, \& S. Wineburg (Eds.), Knowing, teaching and learning history: National and international perspectives (pp. 437-470). New York: New York University Press.

Britt, M. A., Rouet, J.-F., \& Perfetti, C. A. (1996). Using hypertext to study and reason about historical evidence. In J.-F. Rouet, J. J. Levonen, A. P. Dillon, \& R. J. Spiro (Eds.), Hypertext and cognition (pp. 4372). Mahwah, NJ: Erlbaum.
Charney, D. (in press). The impact of hypertext on processes of reading and writing. In S. J. Hilligoss \& C. L. Selfe (Eds.), Literacy and computers. New York: Modern Language Association.

FANG, X., \& SAlvendy, G. (2000). Keyword comparison: A user-centered feature for improving Web search tools. International Journal of Human-Computer Studies, 52, 915-931.

Foltz, P. (1996). Comprehension, coherence, and strategies in hypertext and linear text. In J.-F. Rouet, J. J. Levonen, A. Dillon, \& R. J. Spiro (Eds.), Hypertext and cognition (pp. 109-136). Mahwah, NJ: Erlbaum.

HARP, S. F., \& MAYER, R. E. (1998). How seductive details do their damage: A theory of cognitive interest in science learning. Journal of Educational Psychology, 90, 414-434.

Johnson, H. M., \& Seifert, C. M. (1994). Sources of the continued influence effect: When misinformation in memory affects later inferences. Journal of Experimental Psychology: Learning, Memory, \& Cognition, 20, 1420-1436.

Johnson, H. M., \& SeIfert, C. M. (1999). Modifying mental representations: Comprehending corrections. In H. van Oostendorp \& S. R. Goldman (Eds.), The construction of mental representations during reading (pp. 303-318). Mahwah, NJ: Erlbaum.

LANDOW, G. P. (1989). Hypertext in literary education, criticism, and scholarship. Computers \& the Humanities, 23, 173-198.

MAYER, R. E., Heiser, J., \& LoNn, S. (2001). Cognitive constraints on multimedia learning: When presenting more material results in less understanding. Journal of Educational Psychology, 93, 187-198.

Myers, J. L., \& O'Brien, E. J. (1998). Accessing the discourse during reading. Discourse Processes, 26, 131-157.

Myers, J. L., O'Brien, E. J., Albrecht, J. E., \& Mason, R. A. (1994). Maintaining global coherence. Journal of Experimental Psychology: Learning, Memory, \& Cognition, 20, 876-886.

Perfetti, C. A., Rouet, J.-F., \& Britt, M. A. (1999). Towards a theory of documents representation. In H. van Oostendorp \& S. R. Goldman (Eds.), The construction of mental representations during reading (pp. 99-122). Mahwah, NJ: Erlbaum.

Rouet, J.-F., Britt, M. A., Mason, R. A., \& Perfetti, C. A. (1996), Using multiple sources of evidence to reason about history. Journal of Educational Psychology, 88, 478-493.

Rouet, J.-F., Favart, M., Britt, M. A., \& Perfetti, C. A. (1997). Studying and using multiple documents in history: Effects of discipline expertise. Cognition \& Instruction, 15, 85-106.

Rouet, J.-F., \& LeVonen, J. J. (1996). Studying and learning with hypertext: Empirical studies and their implications. In J.-F. Rouet, J. J. Levonen, A. P. Dillon, \& R. J. Spiro (Eds.), Hypertext and cognition (pp. 9-23). Mahwah, NJ: Erlbaum.

van Oostendorp, H., \& Bonebakker, C. (1999). Difficulties in updating mental representations during reading news reports. In $\mathrm{H}$. van Oostendorp \& S. R. Goldman (Eds.), The construction of mental representations during reading (pp. 319-339). Mahwah, NJ: Erlbaum.

WILEY, J. (2001). Supporting understanding through task and browser design. In J. D. Moore \& K. Stenning (Eds.), Proceedings of the Twenty-Third Annual Conference of the Cognitive Science Society (pp. 1164-1169). Hillsdale, NJ: Erlbaum.

WinEburg, S. S. (1991). Historical problem solving: A study of the cognitive processes used in the evaluation of documentary and pictorial evidence. Journal of Educational Psychology, 83, 73-87.

WRIGHT, P. (1991). Cognitive overheads and prostheses: Some issues in evaluating hypertexts. In R. Furuta \& D. Stotts (Eds.), Proceedings of the Third ACM Conference on Hypertext (pp. 1-12). New York: ACM Press.

(Manuscript received November 15, 2001; revision accepted for publication March 26, 2002.) 